\title{
Secukinumab, Pituitary Enlargement and Panhypopituitarism: Are They Related?
}

\author{
Diogo Ramalho ${ }^{1,}$ André Araújo², Gustavo Rocha ${ }^{3}$, Filipa Duarte-Ribeiro ${ }^{4}$ \\ ${ }^{1}$ Endocrinology Department, Centro Hospitalar de Vila Nova de Gaia/Espinho, Vila Nova de Gaia, Portugal \\ ${ }^{2}$ Neuroradiology Department, Centro Hospitalar de Vila Nova de Gaia/Espinho, Vila Nova de Gaia, Portugal \\ ${ }^{3}$ Endocrinology Department, Centro Hospitalar de Vila Nova de Gaia/Espinho, Vila Nova de Gaia, Portugal \\ ${ }^{4}$ Internal Medicine Department, Centro Hospitalar de Vila Nova de Gaia/Espinho, Vila Nova de Gaia, Portugal
}

\section{Doi: 10.12890/2021_003099 - European Journal of Case Reports in Internal Medicine - @ EFIM 2029}

Received: 01/12/2021

Accepted: $10 / 12 / 2021$

Published: 31/12/2021

How to cite this article: Ramalho D, Araújo A, Rocha G, Duarte-Ribeiro F. Secukinumab, pitituary enlargement and panhypopituitarism: are they related? EJCRIM 2021;8: doi:10.12890/2021_003099.

Conflicts of Interests: The authors declare there are no competing interests.

This article is licensed under a Commons Attribution Non-Commercial 4.0 License

\section{ABSTRACT}

Pituitary adenomas are the most common cause of hypopituitarism associated with pituitary enlargement, but other aetiologies have been emerging, namely immune checkpoint inhibitor-induced hypophysitis (ipilimumab, nivolumab and pembrolizumab). Secukinumab is a recently approved human monoclonal antibody used for the treatment of psoriasis, with no know reported cases of hypophysitis. We describe a challenging case of panhypopituitarism in a patient with a pituitary incidentaloma and a temporal relationship between secukinumab initiation and the manifestation of clinical features suggestive of hypopituitarism. In such intricate work-up, the differential diagnoses should be carefully considered, taking into account the therapeutic and prognostic implications.

\section{LEARNING POINTS}

- Pituitary adenomas are the leading cause of hypopituitarism associated with pituitary enlargement, but clinicians should be aware of non-tumoural causes such as hypophysitis.

- Drug-induced hypophysitis has been described with immune checkpoint inhibitors used for diverse types of malignancies, but there is no evidence of an association between hypophysitis and the novel antipsoriatic agent, secukinumab.

- The differential diagnosis of hypopituitarism requires careful investigation so that management is appropriate and prognosis is improved.

\section{KEYWORDS}

Pituitary neoplasms, hypophysitis, hypopituitarism, secukinumab

\section{INTRODUCTION}

Pituitary enlargement can be due to a wide variety of causes which are often challenging to identify by imaging. Distinguishing them is critical as there are therapeutic and prognostic implications ${ }^{[1]}$. Pituitary adenomas are benign neoplasms of the anterior pituitary. They are the leading cause of intracranial tumours, accounting for up to $10 \%{ }^{[2]}$.

Hypophysitis is the term given to a diverse group of rare inflammatory disorders which primarily or secondarily (systemic conditions) involve the pituitary gland and infundibulum. Immunotherapy is the leading cause of drug-induced hypophysitis, namely agents used to treat metastatic malignancies such as ipilimumab (anti-cytotoxic T lymphocyte-associated antigen-4 (CTLA-4) antibody), nivolumab and pembrolizumab (anti-programmed cell death protein-1 (PD-1) antibodies) ${ }^{[3]}$. 
Secukinumab is an anti-interleukin-17 (IL-17) human monoclonal antibody approved in 2015 by the US Food and Drug Administration and the European Medicines Agency for the treatment of psoriasis. It has been used with proven efficacy and minimal side effects (nasopharyngitis, headache, diarrhoea) ${ }^{[4]}$.

Herein, we describe an incidental finding of pituitary enlargement and panhypopituitarism in a patient treated with secukinumab.

\section{CASE DESCRIPTION}

We present the case of a 66-year-old man treated for 3 years with secukinumab for plaque psoriasis. Since the beginning of treatment, the patient had demonstrated marked fluctuations in mood and in the last year, episodes of delirium, confusion, and a total weight loss of 10 kg. He was admitted to the Emergency Room due to abdominal pain and distension and progressive functional deterioration in the last 3 months. Alcohol consumption and risky behaviour were excluded. At the physical examination, the patient was confused and exhibited mild psoriatic plaques and signs of portal hypertension, namely ascites and caput medusae. Neurological examination was unremarkable, and visual impairment was not confirmed. Blood analysis evidenced pancytopenia, and unaltered ionogram and liver function tests (Table 1). On abdominal ultrasound, mild hepatomegaly and ascites were demonstrated. A cranial computed tomography (CT) scan was normal.

\begin{tabular}{|c|c|c|}
\hline Parameters & Patient value & Reference range \\
\hline Haemoglobin (g/dl) & 7.1 & $13.0-18.0$ \\
\hline Mean haemoglobin concentration (g/dl) & 32 & $32-36$ \\
\hline Red blood cell count (erythrocytes $\left.\times 10^{6} / \mu l\right)$ & 2.53 & 4.4-5.9 \\
\hline Mean cell volume (fl) & 87 & $80-100$ \\
\hline Mean corpuscular haemoglobin (pg) & 31 & $26-34$ \\
\hline Neutrophil count (neutrophils $\times 10^{3} / \mu l$ ) & 1.12 & $3.8-10.6$ \\
\hline Platelet count (platelets $\times 10^{3} / \mathrm{\mu l}$ ) & 123 & $150-440$ \\
\hline Sodium (mmol/l) & 138 & $136-145$ \\
\hline Potassium (mmol/l) & 4.0 & $3.5-5.0$ \\
\hline Chlorine (mmol/l) & 102 & $98-107$ \\
\hline Alanine aminotransferase (U/I) & 18 & $4-50$ \\
\hline Aspartate aminotransferase (U/I) & 36 & $4-33$ \\
\hline Gamma-glutamyl transferase (U/I) & 16 & $5-61$ \\
\hline Alkaline phosphatase (U/I) & 66 & $40-129$ \\
\hline Total bilirubin (mg/dl) & 0.50 & $0.1-1.1$ \\
\hline Albumin (g/dl) & 3.3 & $3.4-4.8$ \\
\hline Total proteins (g/dl) & 5.7 & $6.4-8.3$ \\
\hline International Normalized Ratio & 1.29 & $0.8-1.1$ \\
\hline Glucose $(\mathrm{mg} / \mathrm{dll})^{*}$ & 74 & $60-100$ \\
\hline Adrenocorticotropic hormone $(\mathrm{pg} / \mathrm{ml})^{*}$ & 9.0 & $0-46$ \\
\hline 8 a.m. cortisol $(\mu \mathrm{g} / \mathrm{ml})^{*}$ & 2.3 & $6.2-19.4$ \\
\hline Thyroid-stimulating hormone $(\mu \mathrm{lU} / \mathrm{ml})^{*}$ & 1.19 & $0.27-4.20$ \\
\hline Free T4 $(\mathrm{ng} / \mathrm{ml})^{*}$ & 0.66 & $0.93-1.70$ \\
\hline Prolactin $(\mathrm{ng} / \mathrm{ml})^{*}$ & 21.3 & $4.0-15.2$ \\
\hline Insulin-like growth factor $1(\mathrm{ng} / \mathrm{ml})^{*}$ & 17 & $69-200$ \\
\hline Total testosterone $(\mathrm{ng} / \mathrm{ml})^{*}$ & $<0.02$ & $2.8-8.0$ \\
\hline Free testosterone $(\mathrm{ng} / \mathrm{ml})^{*}$ & $<0,13$ & $4.90-21.60$ \\
\hline Follicle-stimulating hormone $(\mathrm{m} / \mathrm{U} / \mathrm{ml})^{*}$ & 1.89 & $1.5-12.4$ \\
\hline Luteinizing hormone $(\mathrm{mlU} / \mathrm{ml})^{*}$ & 1.00 & $1.7-8.6$ \\
\hline Sex hormone binding globulin (nmol/l)* & 173.6 & $10.0-80.0$ \\
\hline
\end{tabular}

Table 1. Laboratory evaluation during the patient's hospital stay

${ }^{*}$ Requested after alterations seen onpituitary magnetic resonance imaging 
Due to persistence of the confusional state and after exclusion of hepatic encephalopathy, pituitary magnetic resonance imaging (MRI) was performed, which revealed a diffusely enlarged pituitary gland (Figs. 1 and 2). Visual fields were normal. Signs or symptoms suggestive of pituitary dysfunction were difficult to assess because of the altered mental status. Panhypopituitarism was demonstrated by blood tests (Table 1). Hormonal supplementation was started (hydrocortisone $100 \mathrm{mg}$ every 8 hours and levothyroxine $0.05 \mu \mathrm{g} / \mathrm{day}$, both intravenously), which improved the patient's functional status.

A differential diagnosis of panhypopituitarism due to a pituitary macroadenoma or secukinumab-induced hypophysitis (SIH) was assumed. The patient was discharged with hydrocortisone $40 \mathrm{mg}$ in the morning plus $20 \mathrm{mg}$ in the afternoon by mouth and levothyroxine $50 \mathrm{\mu g} / \mathrm{day}$ by mouth, and secukinumab was suspended.

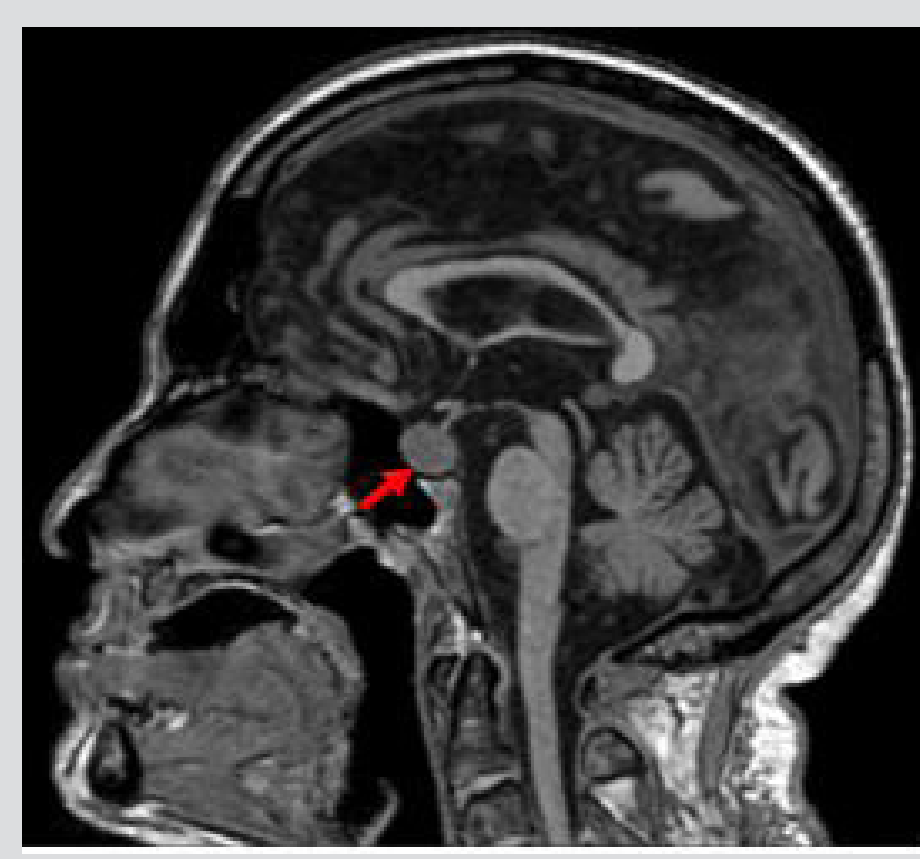

Figure 1. Sagittal T1-weighted image showing a diffusely hypointense enlarged pituitary gland (red arrow). Normal posterior pituitary hyperintensity was present (not shown)

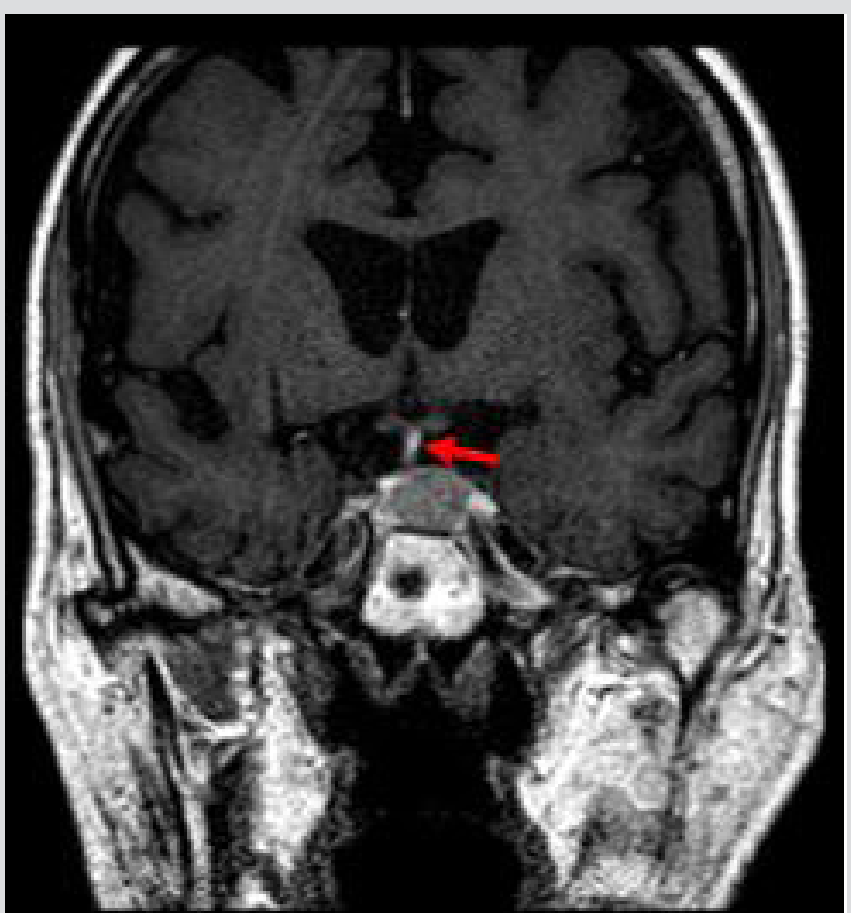

Figure 2. Coronal postcontrast T1-weighted image exhibiting a hypointense pituitary lesion relative to the adenohypophysis, asymmetric, with $14 \times 12 \times 14 \mathrm{~mm}(C \mathrm{C} \times \mathrm{TR} \times \mathrm{AP})$ maximal diameters, causing mild right lateral deviation of the pituitary stalk (red arrow); it insinuates into the left cavernous sinus. Imaging findings are suggestive of macroadenoma

\section{DISCUSSION}

Many new biologic drugs have been emerging for use in psoriasis, namely secukinumab ${ }^{[4]}$. As far as is known, there are no reported cases of $\mathrm{SIH}$. In contrast, immunotherapeutic drugs (ipilimumab, nivolumab and pembrolizumab) have immune-based effects, which often lead to the development of other endocrine immune-related adverse events, such as hypophysitis, adrenal insufficiency and hypothyroidism. The underlying mechanisms are still not known, but growing evidence suggests that adverse eventsmay be related to the initiation of a cascade of responses involving altered self-reactive effector T cells, pituitary inflammation and destruction, and discharge of pituitary self-antigens, leading to the development of more pituitary-reactive effector T lymphocytes $\left.{ }^{[3}\right]$.

T helper 17 (Th17) cells are the major secretors of IL-17, a cytokine with anti-infective properties. However, its excessive production leads to chronic inflammation, and subsequent autoimmune-related disturbances. Thus, it has been recognized as a desirable therapeutic target, such as by secukinumab for psoriasis ${ }^{[5]}$.

Conversely, Hueber et al. ${ }^{[6]}$ reported that using secukinumab to treat inflammatory bowel disease (IBD) was ineffective and led to disease exacerbation, inferring that some Th17 cell populations may be involved in anti-inflammatory functions. The authors postulated that IL-17 blockade may impede a protective function of IL-17 in the bowel. 
Ramos-Levi et al. ${ }^{[7]}$ reported a unique case of an autoimmune hypophysitis in a 35-year-old man who underwent 4-month therapy with ustekinumab for plaque psoriasis. The authors postulated that ustekinumab-induced hypophysitis ensued due to altered balance between pathogenic and non-pathogenic Th17 cells. Ustekinumab changes the levels of IL-23, a cytokine that plays a key role in the differentiation of Th17 cells. Immune analysis demonstrated a comparable degree of IL-17 expression both in Th17 infiltrating cells and in normal pituitary cells. The latter do not secret IL-17. Consequently, the authors proved a cause-and-effect relationship between ustekinumab and hypophysitis. As in the aforementioned case report, we primarily considered the diagnosis of SIH as the patient deteriorated shortly after the beginning of treatment with the monoclonal antibody, and possible interference with protective anti-inflammatory IL-17 functions. The other main aetiologies of hypophysitis may be ruled out, including psoriasis itself, as the disease had been stable after the initiation of secukinumab, together with the absence of a temporal correlation between clinical features of psoriasis and hypophysitis manifestations, and the lack of other autoimmune disorders. However, immune-histopathological work-up was not performed, and hypophysitis could not be confirmed. Hypophysitis diagnosis requires neurosurgical biopsy or resection of sellar or suprasellar tissue. We considered that the risk of significant morbidity to this particular patient, along with the impractical nature of the technique, outweighed the benefit of providing a definite diagnosis.

Gadolinium-enhanced pituitary MRI is the gold-standard method to describe pituitary anatomy. No single radiological feature can clearly discriminate hypophysitis from pituitary adenomas ${ }^{[3]}$. Gutenberg et al. ${ }^{[1]}$ developed a radiological scoring system to distinguish pituitary adenoma from hypophysitis and avoid undue surgery. Our patient scored 1 point, favouring a diagnosis of adenoma, although such a borderline value raises doubts about this diagnosis (Table 2).

\begin{tabular}{|l|c|c|}
\hline Features & Score* $^{*}$ & Patient score \\
\hline Age ( $\leq 30$ years) & -1 & 0 \\
\hline Associated with pregnancy (Yes) & -4 & 0 \\
\hline Pituitary volume ( $\geq 6 \mathrm{~cm} 3$ ) & 2 & 0 \\
\hline Gadolinium enhancement type (medium or high) & -1 & 0 \\
\hline Gadolinium enhancement features (heterogenous) & 1 & 0 \\
\hline Symmetry (asymmetric) & 3 & 3 \\
\hline Posterior pituitary bright spot (lost) & -2 & -2 \\
\hline Stalk size (enlarged) & -5 & 0 \\
\hline Mucosal thickening (present) & 2 & 0 \\
\hline Score (points) & -13 to 8 & 1 \\
\hline
\end{tabular}

Table 2. Radiological scoring system to differentiate autoimmune hypophysitis from non-secreting pituitary adenoma

In light of the clinical, analytical and radiographic findings, including the radiological score ${ }^{[1]}$, we consider non-functioning pituitary macroadenoma to be the most likely diagnosis.

Management of non-functioning pituitary macroadenoma includes active follow-up, transsphenoidal surgery, or radiotherapy. Clinical decisions on treatment approaches should be tailored to the patient, depending on the clinical setting and patient preference. In patients with no visual impairment, a conservative approach that includes careful medical follow-up (hormonal, imaging and neuro-ophthalmological evaluation) may be reasonable ${ }^{[8]}$. This option was suitable for our patient as his deteriorated status impeded a surgical approach.

We consider this case report as a landmark regarding the differential diagnosis of panhypopituitarism. The rare coincidence of a temporal correlation between the initiation of secukinumab and the emergence of clinical manifestations, and the unclear radiological findings (including the borderline clinicoradiological score ${ }^{[1]}$ ) led us to a complicated diagnostic investigation.

Although secukinumab may interfere with anti-inflammatory IL-17 function in the bowel ${ }^{[6]}$, as far as the authors know, no studies have investigated a similar role in the pituitary gland. Hence, further research is required in this field. 


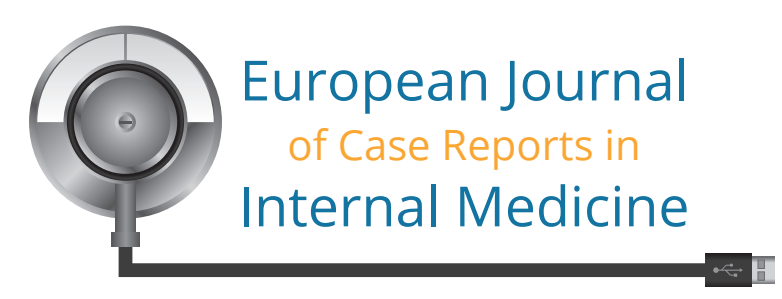

\section{REFERENCES}

1. Gutenberg A, Larsen J, Lupi I, Rohde V, Caturegli P. A radiologic score to distinguish autoimmune hypophysitis from nonsecreting pituitary adenoma preoperatively. AJNR Am J Neuroradiol 2009;30(9):1766-1772.

2. Freda PU, Beckers AM, Katznelson L, Molitch ME, Montori VM, Post KD, et al. Pituitary incidentaloma: an Endocrine Society clinical practice guideline. J Clin Endocrinol Metab 2011;96(4):894-904.

3. Joshi MN, Whitelaw BC, Carroll PV. MECHANISMS IN ENDOCRINOLOGY: Hypophysitis: diagnosis and treatment. Eur J Endocrinol 2018;179(3):R151-R163.

4. Yang EJ, Beck KM, Liao W. Secukinumab in the treatment of psoriasis: patient selection and perspectives. Psoriasis (Auckl) 2018;8:75-82.

5. Beringer A, Noack M, Miossec P. IL-17 in chronic inflammation: from discovery to targeting. Trends Mol Med 2016;22(3):230-241.

6. Hueber W, Sands BE, Lewitzky S, Vandemeulebroecke M, Reinisch W, Higgins PD, et al. Secukinumab, a human anti-IL-17A monoclonal antibody, for moderate to severe Crohn's disease: unexpected results of a randomised, double-blind placebo-controlled trial. Gut 2012;61(12):1693-1700.

7. Ramos-Levi AM, Gargallo M, Serrano-Somavilla A, Sampedro-Nunez MA, Fraga J, Marazuela M. Hypophysitis following treatment with ustekinumab: radiological and pathological findings. Front Endocrinol (Lausanne) 2018;9:83.

8. Esposito D, Olsson DS, Ragnarsson O, Buchfelder M, Skoglund T, Johannsson G. Non-functioning pituitary adenomas: indications for pituitary surgery and post-surgical management. Pituitary 2019;22(4):422-434. 